\title{
Effects of Linear and Non-periodized Combined Training on Health-related Quality of Life of Adults With Obesity: a Randomized Clinical Trial
}

Willen Remon Tozetto ( $\nabla$ willen_tozetto@hotmail.com )

Universidade Federal de Santa Catarina https://orcid.org/0000-0001-8856-1678

Larissa dos Santos Leonel

Federal University of Santa Catarina: Universidade Federal de Santa Catarina

Tiago Turnes

Federal University of Santa Catarina: Universidade Federal de Santa Catarina

Giovani Firpo Del Duca

Federal University of Santa Catarina: Universidade Federal de Santa Catarina

\section{Research}

Keywords: Endurance Training, Exercise, Mental Health, Obesity, Weight Reduction Programs, Resistance Training

Posted Date: April 19th, 2021

DOI: https://doi.org/10.21203/rs.3.rs-412559/v1

License: (c) (i) This work is licensed under a Creative Commons Attribution 4.0 International License. Read Full License 


\section{Abstract}

Background: Health-related quality of life (HRQoL) is impaired by obesity and can be improved by combined training, even the effects of training periodization on HRQoL are not clear. The study aimed to compare combined training with the non-periodized and the linear periodization on HRQoL of obese adults.

Methods: This is a blinded, controlled clinical trial involving adults with obesity (BMI $\left.\geq 30 \mathrm{~kg} / \mathrm{m}^{2}\right)$, randomized into control (CG), non-periodized (NG) and linear periodization (PG) group. Three times a week for 16 weeks, NG and PG performed combined training for 60 minutes. The NG performed aerobic exercises between $50-59 \%$ of the reserve heart rate (HRres) and strength at 10-12 maximum repetitions (MR). The PG started with $40-49 \%$ of HRres and 12-14 MRs and progressively increased the intensity (50$59 \%$ and $10-12 \mathrm{MRs} ; 60-69 \%$ and 8-10 MRs) with total volume equalized with NG. HRQoL was investigated through the SF-36 questionnaire, according to its components and domains. In statistics, Generalized Estimation Equations and mean differences $(\Delta)$ were used.

Results: Of the 69 participants ( 23 in each group), 36 completed the intervention (CG=13, NG=9 and $P G=14)$. A significant difference was observed in time of physical function, with superiority in training groups (CG: $\Delta=1.2$ vs NG and PG, respectively: $\Delta=10.0$ ). The mental component and mental health domain showed a significant difference for $\mathrm{NG}(\Delta=30.2$ and $\Delta=23.1$, respectively).

Conclusion: The combined training was able to bring benefits to the physical functioning of adults with obesity and, specifically, the non-periodized training improved mental health indicators effectively.

Name of the registry: Effects of different types of Aerobic Exercise and Weight Training protocols combined on adult health indicators with Obesity.

Trial registration number: RBR-3c7rt3

Date of registration: $07 / 02 / 2018$

URL of trial registry record: https://ensaiosclinicos.gov.br/rg/RBR-3c7rt3

\section{Introduction}

Obesity is one of the main Chronic Non-Communicable Diseases (CNCD) in the modern world, caused by the positive energy balance and raised from multiple factors such as inadequate eating habits, low levels of physical activity and a stressful lifestyle [1]. It is a global epidemic [2], reaching $13 \%$ of the world population [1] and $18.9 \%$ of the Brazilian population [3]. The increase in its prevalence has direct consequences on the Health-Related Quality of Life (HRQoL) [4-6], together with increased morbidity and mortality from the disease [7]. 
Excess body fat is closely linked to physical and psychosocial losses, with a dose-response effect on HRQoL $[8,9]$. The disease can cause functional limitations, reduced work capacity and subsequent psychological problems with increased anxiety, obsessive-compulsive disorder, paranoid personality disorder and depression $[10,11]$. In order to mitigate the damage caused by obesity and improve HRQoL, several strategies are used, with regular physical exercise and training being one of the most effective forms of treatment for improving various health parameters in this population $[7,12]$.

Among the numerous methods available, combined training, which combines aerobic and muscle strength exercises, seems to more effectively attenuate the losses resulting from obesity when compared to training performed in isolation [13], with greater benefits in physical and mental health [7, 12, 14], which could contribute to the improvement of HRQoL. In addition, progression in training, especially in the intensity of exercise, shows superiority in improving health parameters related to obesity compared to training without progression, such as improving the metabolic profile, reducing chronic pain, releasing monoamines, distracting negative thoughts and consequent adherence to training, contributing to physical and psychological well-being [5, 15-18]. However, lower intensities provided greater gains in improving HRQoL [19] although not unanimously [20]. Thus, it is not clear in the literature the effects of intensity progression during combined training on HRQol. Studies are only concerned with comparing combined training with aerobic and strength training alone and not with different periods [21-23].

Therefore, the effects of the intensity of combined physical exercises are still scarce in relation to HRQoL of populations with CNCD's, such as obesity. It is hypothesized that the increase in structured intensity during combined training may provide greater benefits on HRQoL in people with obesity [21-23], since it enhances other parameters related to the health of this population [5, 15-17]. Still, moderate or high intensities may initially cause greater discomfort for obese beginners, such as joint pain and excessive tiredness [16]. Thus, initially adopting lower intensities could indirectly improve the perception of HRQoL in this population. Therefore, the aim of this study was to compare the effects of 16 weeks of combined training with non-periodized and linear periodization in the domains and components of Health-Related Quality of Life of obese adults.

\section{Methods}

\section{Study design}

A blind randomized controlled trial with three groups of obese individuals. Participants performed a series of assessments and answered the SF-36 questionnaire for HRQoL analysis before and after a 16-week intervention period, in which they were divided into three groups: control group (CG), non-periodized group (NG) and linear periodization group (PG). For this purpose, volunteers residing in the metropolitan area of Florianópolis, Santa Catarina state, Brazil were recruited. Methodological details can be found in the study protocol article [24]. This study is approved by the Human Research Ethics Committee (2.448.674) and registered in the Brazilian Registry of Clinical Trials (RBR-3c7rt3).

\section{Participants}


We selected men and women aged 20 to 50 years with Body Mass Index (BMI) between $30-39.9 \mathrm{~kg} / \mathrm{m}^{2}$ (degree obesity I and II). Those who did not exercise weekly more than twice in the past three months, were non-smokers, did not consume excessive alcohol ( $\geq 7$ drinks and $\geq 14$ weekly drinks for women and men, respectively) [25], had no osteomioarticular pathology limiting practice of physical exercises, were not on medication to control and/or treat obesity, underwent no weight-loss surgical procedures were eligible for this study and who had other diseases besides obesity. Participants who manifested formal dropout, who did not answer the proposed questionnaires or who changed the habits observed in the eligibility criteria at the end of the study were excluded.

After signing the free and informed consent form, all participants underwent a series of evaluations. Then, the allocation was stratified by gender, age, and BMI, collected at the baseline, with a ratio of 1:1:1 through an online platform (www.randomized.org). Independent researchers not involved in the evaluations and intervention conducted this process. The allocation list was unveiled to the coaches only on the intervention's starting date. All study procedures took place from March to November 2018.

\section{Interventions}

PG and NG participated in 16 weeks of combined training (aerobics and muscle strength in the same session). Aerobics training was performed continuously by walking and/or running on the athletic track, with intensities prescribed as per the percent reserve heart rate ranges (\%HRres). Strength training was performed in multiple sets using 6 exercises involving the large muscle groups in the following order: straight bench press, chest machine fly, low row, pulldown, barbell squat, and leg press $45^{\circ}$, with prescription for maximum repetition zones (MR). The established weekly frequency was 3 nonconsecutive times, and no criteria were established for the exclusion of participants in case of low adherence. The training lasted an average of 60 minutes, the first half being dedicated to aerobic training. . The PG participated in a linear periodization training, divided into three mesocycles of five weeks each, progressing between the light intensities (40-49\% HRres/12-14 MR), moderate (50-59\% HRres/10-12 MR) and vigorous (60-69\% HRres/8-10 MR). In the NG, intensity remained moderate (50-59\% HRres/10-12 MR) throughout the study. The first week was used for training familiarization for both groups (30-39\% HRres/10-15 MR). The CG did not receive any intervention and was instructed to maintain its routine activities.

\section{Assessments for sample characterization and exercise prescription}

Before and after the intervention period, the participants filled in an online questionnaire on sociodemographic information, containing: sex (male and female), marital status (with and without partner), ethnicity (white or others), education (in years of study) and age (in complete years). Body composition was assessed using tetrapolar electrical bioimpedance (In Body 720, Ottoboni, Rio de Janeiro, Brazil), handled by experienced evaluators who followed the Brazilian Association of Nutrology guidelines [26]. To prescribe aerobic training by HRres, the maximum and resting heart rates were used to calculate the ideal training zone, which were obtained using portable heart rate meters (Polar $\left.{ }^{\circledR}, \mathrm{S} 810 \mathrm{i}\right)$. The maximum heart rate was derived by the incremental test described by Libardi et al. [14]. The resting 
heart rate was measured while the participant was lying down with the frequency meter strap positioned. Three one-minute notes were made with a one-minute interval between them. The reference value was the average of the measurements observed at different moments. Resting heart rate reassessments were performed at the end of each mesocycle to adjust the intensity.

\section{Outcomes assesment}

The HRQoL was measured using the SF-36 questionnaire developed by Ware and Sherbourne, with the version presented to the participants was translated and validated in Portuguese by Ciconelli et al [27]. Each participant filled in the questionnaire through the online filling in platform Question Pro® preintervention and soon after the end of 16 weeks. This questionnaire evaluates the HRQoL through 36 questions involving eight domains to evaluate separately each aspect of the construct. These domains are analyzed according to the Physical and Mental Component Summary, but the individual use of the domains is emphasized to better understand the responses. The Physical Component Summary (PCS) is divided into role-physical, physical functioning, bodily pain and general health status. On the other hand, the Mental Component Summary (MCS) is separated into role-emotional, social functioning, mental health and vitality. Its scale ranges from 0 to 100, with higher values representing better HRQoL within each domain or component.

\section{Statistical Analysis}

Sample calculation was performed using GPower® 3.1.7 software, adopting a significance level of 0.05 , a power of $80 \%$, and an effect size of 0.18 in repeated measures analysis, with a ratio of 1:1:1 among the three study groups for the main variable of the main project, i.e. $\mathrm{VO}_{2 \max }$ [24]. The calculation yielded a minimum of 26 participants in each group, totaling at least 78 participants.

To characterize the sample, sociodemographic variables were used. Continuous variables were expressed as the mean and standard error and categorical variables as relative frequency. Baseline differences between groups were tested using analysis of variance for independent samples (one-way ANOVA) and chi-square $\left(\chi^{2}\right)$. Data distribution was verified using the Shapiro-Wilk.

Those participants' HRQol was analyzed who stayed until the end of the study and had all the evaluation data. Intra and intergroup analyses were performed using generalized estimation equations (GEE) with post-hoc Bonferroni. Additional analysis with intention to treat is presented in the supplementary material. Data were expressed as mean and standard error, with $a=0.05$. Effect size analyses were performed using partial eta-squared $\left(\eta_{\mathrm{p}}^{2}\right)$, considering for interpretation as: small $(\leq 0.13)$, medium $(0.14$ $\geq \eta_{\mathrm{p}}^{2} \leq 0.25$ ) and large ( $\geq 0.26$ ) [28]. The magnitude of (post-pre) difference between the evaluations was expressed as the mean difference $(\Delta)$. All these analyses were performed using IBM SPSS version 21.0 (IBM Corp., Armonk, NY, EUA). Graphpad PRISM 7 was used to illustrate the scores of each participant's physical and mental component in the pre and post intervention moments. 


\section{Results}

The study was attended by 515 volunteers willing to participate. However, after considering the eligibility criteria, 69 remained, who were randomized into three groups (Control group: 23; Non-periodized group: 23; Periodized group: 23). A total of 36 completed all phases of the trial $(C G=13, N G=9$ and $P G=14)$, and were included in the analyses. With this sample, for the variables of the PCS and MCS, the effect size of Cohen's $f$ was 0.24 and 0.44 , offering a sampling power of $70 \%$ and $99 \%$, respectively. Figure 1 shows the details of this information.

Table 1 represents the comparison of sociodemographic characteristics of the obese adults whose data were analyzed. No statistically significant differences were found between the groups according to the analyzed variables.

Table 1

Characteristics of participant who completed the trial $(n=36)$.

\begin{tabular}{|c|c|c|c|c|}
\hline \multirow[t]{2}{*}{ Variable } & $\begin{array}{l}\text { Control } \\
(n=13)\end{array}$ & $\begin{array}{l}\text { Non-periodized } \\
(n=9)\end{array}$ & $\begin{array}{l}\text { Periodized } \\
(n=14)\end{array}$ & $\mathrm{p}$ value \\
\hline & n (\%) & n (\%) & n (\%) & \\
\hline Sex (female) & $8(36.4 \%)$ & $6(27.3 \%)$ & $8(36.4 \%)$ & 0.900 \\
\hline Marital status (with partner) & $10(38.5 \%)$ & $7(26.9 \%)$ & $9(34.6 \%)$ & 0.697 \\
\hline \multirow[t]{2}{*}{ Ethnicity (white) } & $11(37.9 \%)$ & $7(24.1 \%)$ & $11(37.9 \%)$ & 0.897 \\
\hline & $\overline{\mathbf{x}}( \pm \mathrm{SE})$ & $\overline{\mathbf{x}}( \pm \mathrm{SE})$ & $\overline{\mathbf{x}}( \pm \mathrm{SE})$ & \\
\hline Age (years) & $35.2 \pm 2.1$ & $37.4 \pm 1.3$ & $38.4 \pm 1.6$ & 0.388 \\
\hline Education (years) & $15.5 \pm 0.5$ & $15.2 \pm 0.9$ & $16.6 \pm 0.8$ & 0.388 \\
\hline BMI $\left(\mathrm{kg} / \mathrm{m}^{2}\right)$ & $33.1 \pm 0.8$ & $32.3 \pm 0.8$ & $33.2 \pm 0.5$ & 0.739 \\
\hline
\end{tabular}

Participants in the NG and PG groups included in the analyses reached a $67.1 \%$ and $58.6 \%$ frequency of sessions, respectively, with no difference between groups $(p=0.343)$. The frequency for the first mesocycle was $76.3 \%$ and $69.5 \%(p=0.398)$, in the second $63.7 \%$ and $44.8 \%(p=0.113)$ and in the third $59.3 \%$ and $52.4 \%(p=0.476)$ for $N G$ and $P G$, respectively. The prescribed intensity was met throughout the aerobic training sessions by $90 \%$ by the participants, with no differences in the total volume of aerobic training $(p=0.657)$ and strength $(p>0.999)$ between groups.

Table 2 presents the HRQoL components and domains. In the PCS and its domains, a significant increase was observed with medium effect size over time only in physical functioning, with an improvement of 10 points for NG and PG, while CG increased only 1.2 points. Analyzing the differences in the values of role- 
physical and bodily pain, the training groups improved their scores, while the CG worsened. Although expected, the groups that trained, regardless of periodization, seemed to improve the PCS when compared to the CG (with the exception of general health for the PG). Significant interactions were found in the MCS and mental health, reporting improved scores for the NG. Still, significant results over time were observed in all MCS domains, with medium to large effect sizes, with the three groups showing an increase in most MCS domains (with the exception of mental health for PG).

Individual changes, before and after the intervention, are shown in Fig. 2. In the PCS (A) and MCS (D) of the CG, six (43.9\%) and four (69.3\%) participants, respectively, showed improvement in the score. In graph B, six participants $(66.6 \%)$ showed an increase in the PCS score, with the initial score of these individuals being lower than the others. In graph $\mathrm{E}$, the individual with the highest initial score was the only one to reduce his score among the nine participants. In the PG (C; F) representations, nine (64.3\%) people improved their PCS score, while eleven (78.6\%) increased the MCS.

\section{Discussion}

This study aimed at compare the effect of 16 weeks' non-periodized and linear periodization combined training on the sleep quality of obese adults. Regarding the MCS, improvements were found in this component and in the domain of mental health after training only in NG. In addition, greater magnitudes of improvement were found in the MCS domains for the NG compared to the PG and CG, pertinent to the improvement of the psychological and social aspects, however, without statistical difference.

Furthermore, a significant difference in time was observed for the functional capacity domain, with an increase in the score of the groups that underwent training, regardless of periodization.

The benefits of combined exercise on muscle structure, cardiorespiratory fitness, reducing systemic inflammation and metabolic markers in people with obesity are already evident $[7,12,13]$, as well as the reduction of articulate and back pain [18]. Thus, the improvement in functional capacity in the groups that performed the combined training is due to the aforementioned improvements; however, the different training prescriptions did not provide different results in this domain. The benefits of increased intensity, observed in studies with aerobic or strength exercises performed in isolation, [15, 29], may be linked to the different forms of manipulation of the training load, with greater amplitudes than those used in the present study (40-69\% HRres; $14-8 \mathrm{MR})$. This helps to explain the similar improvements observed in some domains PCS for both training groups.

Studies that have looked at the effect of combined training on HRQoL, and included overweight people, have conflicting results. Sillanpää et al., [23] observed an improvement in general health only among PCS, with a tendency to worsen the domains of role-physical and bodily pain in adults. Differently, Goldfield et al., [21] when analyzing the effect of training on adolescents, reported a significant improvement in functional capacity. Whereas, Baptista et al., [30] when evaluating the HRQoL of elderly people, observed improvement in three of the four physical domains (except role-physical), in addition to the PCS itself. It should be noted that the three studies cited used some form of training progression, making it impossible to compare them with studies with non-periodized methodologies. Therefore, the results of training 
interventions combined with and without progression are still inconclusive on their effects on HRQoL PCS.

The regular practice of physical exercises attenuates psychosocial disorders in different ways, either by improving the immune system and other physiological markers or else by the capacity for distraction and self-efficacy [17]. In addition, improvement results in the MCS may precede the physical benefits, as they need greater stimuli for their adaptation, while the feeling of belonging to the group, distraction from stressful environments and a feeling of increased vigor provide psychological well-being, achieved even with reduced training volume [31-33]. Evidence has suggested that exercising 30 to 60 minutes, three to five times a week, reduces mental burden, improving aspects of mental health [32], and in more severe disorders the practice is efficient, for example, improving depressive conditions [34]. It is worth mentioning that the studied population did not have any diagnosed psychological disorder and, even so, it had positive effects on mental health. The improvement of this domain is of paramount importance in this population, and population-based evidence from the same region where the study was conducted pointed to a $45 \%$ increase in the prevalence of depressive disorder when there is the presence of CNCD [35].

In the longitudinal study by Chekroud et al., [32] higher intensities were associated with improved mental health in more than 1.2 million individuals. This corroborates the initial hypothesis that the program with linear periodization would present superior results due to the increase in intensity presenting superiority in other health parameters $[5,7,12,14-16]$. Despite this, Reid et al., [22] when verifying the impact of progressive combined training on patients with type II diabetes mellitus and excess weight, did not report an improvement in MCS, which according to the authors was due to the excessive fatigue of the modality. This corroborates the findings of the present study, since more expressive results were found only in the group with no progression of intensity for mental health and MCS and, even if not significant, in roleemotional, social functioning and vitality. The difference in the frequency of training between the groups could explain the improvement of the MCS domains observed in the NG, but, despite $8.5 \%$ higher than the PG, it did not present significant differences between them, denoting not being a weighting factor for improvement [36]. Another possibility are intrinsic factors linked to the practice of exercises, such as a taste for practice and motivation, exercising a direct influence on health parameters [36], but they represent a limitation of the present study since they were not evaluated.

The applied clinical design is one of the main strengths of this study, being an intervention lasting 16 weeks of training combined with different forms of periodization, using a control group for comparison, with randomization and blinding. Likewise, the equalization of the training volume allows the different periods to be compared in an equivalent manner. It should be highlighted the re-evaluation of the resting HR to adjust the participants' internal load to maintain the proposed intensity. Another important factor for the results is the sample of individuals with only obesity, free of other comorbidities. This reduced the eligible candidates for the study, but increased the representativeness of the HRQoL analysis in individuals with obesity. 
As limitations, both the low adherence of the participants to the training sessions and the number of dropouts from the study must be considered when observing the results. It is likely that the participants in this research would benefit from greater effects by training more often, with consequent implications for HRQoL. Likewise, dropping out of the study may be due to low self-esteem, vitality and other negative psychological factors present in this population [37]. Thus, more expressive results were not found in the PCS, possibly due to the low attendance of the participants to the training, given the need for frequent stimuli for the physiological adaptations to occur. It is likely that dropouts from this research are less aware of their health status, having a negative effect on their motivation [38]. It is important to recognize that satisfaction, taste and pleasure when carrying out training is essential for the maintenance of individuals, therefore, there is the possibility that the training proposal used, without adherence strategies, is not sufficient to motivate this population [36]. There is a possibility that the sample in this study was composed of some people with depressive disorder, or at least with symptoms, due to the low MCS score. The search for professional help and the clinical diagnosis of individuals affected by mental disease tend to take longer to happen, since the perception of symptoms and the perception of worsening health are mediators in the process, [39] not recognizing the presence of these diseases when questioned initially in that study.

\section{Conclusion}

In conclusion, non-periodized combined training is effective in improving mental health and MCS. However, it is not possible to state that the non-periodized model is superior to linear periodization, since similar results were found in the PCS between the training groups. From these results, we recommend that the training prescription combined with non-periodized structure, that is, with periodic adjustments of the internal load to achieve the physical and mental benefits of HRQoL in adults with obesity. Future studies should investigate the relationship between the proposed periodization and the improvement of HRQoL, contributing to greater understanding of the benefits provided in people with obesity, including different forms of periodization, such as the wave. Manipulations in the training frequency, duration and intensity proposed may expand the knowledge about the possible implications in HRQoL. These studies must take into account the low adherence of this population to training, inclusive, adopting strategies to maintain adherence to exercise programs.

\section{Abbreviations}

HRQoL: Health-related quality of life

BMl: Body Index Mass

CG: Control Group

NG: Non-Periodized Group

PG: Linear Periodization Group 
HRres: Reserve Heart Rate

MR: Maximum Repetitions

CNCD: Chronic Non-Communicable Diseases

PCS: Physical Component Summary

MCS: Mental Component Summary

\section{Declarations}

\section{Ethics approval and consent to participate}

The research was approved by the Human Research Ethics Committee of the Federal University of Santa Catarina (2.448.674). All participants were duly informed about the procedures and signed the free and informed consent form.

\section{Consent for publication}

Not applicable.

\section{Availability of data and materials}

The datasets used and/or analysed during the current study are available from the corresponding author on reasonable request.

\section{Competing interests}

The authors declare that they have no competing interests.

\section{Funding}

This study received no funding.

\section{Authors' contributions}

All authors participated with substantial contributions to the design of this manuscript. WRT and LSL participated in the analysis and interpretation of the data, preparation of the article, and critical review of the article and approval of the final version. TT and GFDD contributed to the interpretation of the data, critical review of the article and approval of the final version.

\section{Acknowledgments}

We thank all the participants and researchers who collaborated for the execution of this project. The author WRT thanks the Santa Catarina State Research and Innovation Support Foundation (FAPESC) - 
Brazil, for the grant of the scholarship (Notice n. ${ }^{\circ}$ 007/PPGEF/2017).

\section{Trial Registration}

This study is registered at www.ensaiosclinicos.gov.br/ (No. RBR-3c7rt3)."

\section{References}

1. WHO (2018) Obesity and overweight. In: World Health Organ. http://www.who.int/news-room/factsheets/detail/obesity-and-overweight. Accessed 17 May 2018

2. Turcato TD, Lima CP, Serralta FB (2017) Obesidade, características de personalidade e sofrimento psicológico: um estudo de caso controle. Quad Psicol 19:59-71

3. Brasil (2017) VIGITEL-Vigilância de fatores de risco e proteção para doenças crônicas por inquérito telefônico. 160

4. Hollander EL, Picavet HSJ, Milder IE, Verschuren WMM, Bemelmans WJE, de Groot LCPGM (2013) The impact of long-term body mass index patterns on health-related quality of life: the Doetinchem Cohort Study. Am J Epidemiol 178:804-812

5. Castelli DM, Centeio EE, Hwang J, Barcelona JM, Glowacki EM, Calvert HG, Nicksic HM (2014) VII. The history of physical activity and academic performance research: informing the future. Monogr Soc Res Child Dev 79:119-148

6. Verkleij SPJ, Adriaanse MC, Wendel-Vos GCW, Schuit AJ (2013) Longitudinal relation between weight change and quality of life in a community-based population: a prospective cohort study. Eur $\mathrm{J}$ Public Health 23:285-290

7. Brunelli DT, Chacon-Mikahil M, Gáspari AF, Lopes WA, Bonganha V, Bonfante I, Bellotto ML, Libardi CA, Cavaglieri CR (2015) Combined Training Reduces Subclinical Inflammation in Obese Middle-Age Men. Med Sci Sports Exerc 47:2207-2215

8. Svärd A, Lahti J, Roos E, Rahkonen O, Lahelma E, Lallukka T, Mänty M (2017) Obesity, change of body mass index and subsequent physical and mental health functioning: a 12-year follow-up study among ageing employees. BMC Public Health. https://doi.org/10.1186/s12889-017-4768-8

9. Ul-Haq Z, Mackay DF, Fenwick E, Pell JP (2013) Meta-analysis of the association between body mass index and health-related quality of life among adults, assessed by the SF-36. Obesity 21:E322-327

10. Brandheim S, Rantakeisu U, Starrin B (2013) BMI and psychological distress in 68, 000 Swedish adults: a weak association when controlling for an age-gender combination. BMC Public Health 13:68

11. Martínez EV, Gutiérrez-Bedmar M, García-Rodríguez A, Mariscal A, Muñoz-Bravo C, Navajas JF-C (2014) Weight Status and Psychological Distress in a Mediterranean Spanish Population: A Symmetric U-Shaped Relationship. Nutrients 6:1662-1677

12. Bonfante ILP, Chacon-Mikahil MPT, Brunelli DT, Gáspari AF, Duft RG, Lopes WA, Bonganha V, Libardi CA, Cavaglieri CR (2017) Combined training, FNDC5/irisin levels and metabolic markers in obese 
men: A randomised controlled trial. Eur J Sport Sci 17:629-637

13. Schwingshackl L, Dias S, Strasser B, Hoffmann G (2013) Impact of Different Training Modalities on Anthropometric and Metabolic Characteristics in Overweight/Obese Subjects: A Systematic Review and Network Meta-Analysis. PLoS ONE 8:e82853

14. Libardi CA, Souza GV, Gáspari AF, et al (2011) Effects of concurrent training on interleukin-6, tumour necrosis factor-alpha and C-reactive protein in middle-aged men. J Sports Sci 29:1573-1581

15. Chiu C-H, Ko M-C, Wu L-S, Yeh D-P, Kan N-W, Lee P-F, Hsieh J-W, Tseng C-Y, Ho C-C (2017) Benefits of different intensity of aerobic exercise in modulating body composition among obese young adults: a pilot randomized controlled trial. Health Qual Life Outcomes 15:168

16. Marandi SM, Abadi NGB, Esfarjani F, Mojtahedi H, Ghasemi G (2013) Effects of intensity of aerobics on body composition and blood lipid profile in obese/overweight females. Int J Prev Med 4:S118

17. Mikkelsen K, Stojanovska L, Polenakovic M, Bosevski M, Apostolopoulos V (2017) Exercise and mental health. Maturitas 106:48-56

18. Wasser JG, Vasilopoulos T, Zdziarski LA, Vincent HK (2017) Exercise benefits for chronic low back pain in overweight and obese individuals. PM\&R 9:181-192

19. Gillison FB, Skevington SM, Sato A, Standage M, Evangelidou S (2009) The effects of exercise interventions on quality of life in clinical and healthy populations; a meta-analysis. Soc Sci Med 68:1700-1710

20. Conn VS, Hafdahl AR, Brown LM (2009) Meta-analysis of quality-of-life outcomes from physical activity interventions. Nurs Res 58:175-183

21. Goldfield GS, Kenny GP, Alberga AS, Tulloch HE, Doucette S, Cameron JD, Sigal RJ (2017) Effects of aerobic or resistance training or both on health-related quality of life in youth with obesity: the HEARTY Trial. Appl Physiol Nutr Metab 42:361-370

22. Reid R, Tulloch H, Sigal R, Kenny G, Fortier M, McDonnell L, Wells G, Boule N, Phillips P, Coyle D (2010) Effects of aerobic exercise, resistance exercise or both, on patient-reported health status and well-being in type 2 diabetes mellitus: a randomised trial. Diabetologia 53:632-640

23. Sillanpää E, Häkkinen K, Holviala J, Häkkinen A (2012) Combined Strength and Endurance Training Improves Health-Related Quality of Life in Healthy Middle-Aged and Older Adults. Int J Sports Med 33:981-986

24. Streb AR, da Silva RP, Leonel L dos S, Tozetto WR, Gerage AM, Benedet J, Delevatti RS, Turnes T, Del Duca GF (2019) Comparison of linear periodized and non-periodized combined training in health markers and physical fitness of adults with obesity: Clinical trial protocol. Contemp Clin Trials Commun 15:21

25. Xi B, Veeranki SP, Zhao M, Ma C, Yan Y, Mi J (2017) Relationship of Alcohol Consumption to AllCause, Cardiovascular, and Cancer-Related Mortality in U.S. Adults. J Am Coll Cardiol 70:913-922 26. ABRAN (2015) Avaliação da Composição corporal por Bioimedanciometria. 
27. Ciconelli RM, Ferraz MB, Santos W, Meinão I, Quaresma MR (1999) Tradução para a língua portuguesa e validação do questionário genérico de avaliação de qualidade de vida SF-36 (Brasil SF36). Rev Bras Reum 39:143-50

28. Cohen M, Jaffray J (1988) Is savage's independence axiom a universal rationality principle? Behav Sci 33:38-47

29. Harries SK, Lubans DR, Callister R (2015) Systematic Review and Meta-analysis of Linear and Undulating Periodized Resistance Training Programs on Muscular Strength. J Strength Cond Res 29:1113-1125

30. Baptista LC, Machado-Rodrigues AM, Martins RA (2017) Exercise but not metformin improves healthrelated quality of life and mood states in older adults with type 2 diabetes. Eur J Sport Sci 17:794804

31. Chang Y-C, Lu M-C, Hu I-H, Wu W-Cl, Hu SC (2017) Effects of different amounts of exercise on preventing depressive symptoms in community-dwelling older adults: a prospective cohort study in Taiwan. BMJ Open 7:e014256

32. Chekroud SR, Gueorguieva R, Zheutlin AB, Paulus M, Krumholz HM, Krystal JH, Chekroud AM (2018) Association between physical exercise and mental health in 1.2 million individuals in the USA between 2011 and 2015: a cross-sectional study. Lancet Psychiatry 5:739-746

33. Fanning J, Walkup MP, Ambrosius WT, Brawley LR, Ip EH, Marsh AP, Rejeski WJ (2018) Change in health-related quality of life and social cognitive outcomes in obese, older adults in a randomized controlled weight loss trial: Does physical activity behavior matter? J Behav Med 41:299-308

34. Harvey SB, Øverland S, Hatch SL, Wessely S, Mykletun A, Hotopf M (2017) Exercise and the Prevention of Depression: Results of the HUNT Cohort Study. Am J Psychiatry 175:28-36

35. Boing AF, Melo GR, Boing AC, Moretti-Pires RO, Peres KG, Peres MA (2012) Associação entre depressão e doenças crônicas: um estudo populacional. Rev Saúde Pública 46:617-623

36. Vella CA, Taylor K, Drummer D (2017) High-intensity interval and moderate-intensity continuous training elicit similar enjoyment and adherence levels in overweight and obese adults. Eur J Sport Sci 17:1203-1211

37. Moroshko I, Brennan L, O'Brien P (2011) Predictors of dropout in weight loss interventions: a systematic review of the literature. Obes Rev Off J Int Assoc Study Obes 12:912-934

38. Colombo O, Ferretti VVV, Ferraris C, Trentani C, Vinai P, Villani S, Tagliabue A (2014) Is drop-out from obesity treatment a predictable and preventable event? Nutr J 13:13

39. Koopmans GT, Lamers LM (2007) Gender and health care utilization: The role of mental distress and help-seeking propensity. Soc Sci Med 64:1216-1230

\section{Tables}

Due to technical limitations, table 2 docx is only available as a download in the Supplemental Files section. 


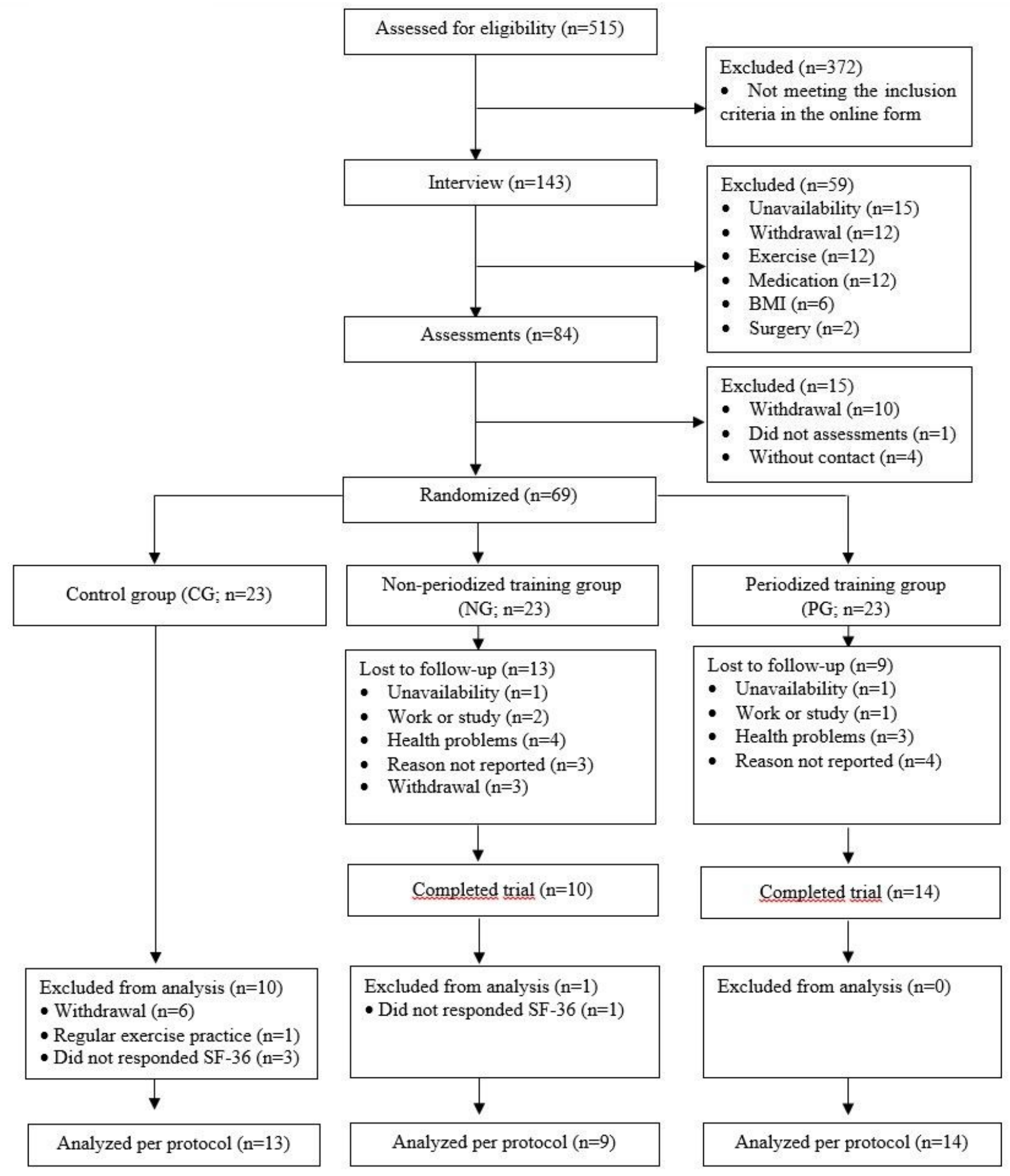

\section{Figure 1}

Flowchart of the study process. 

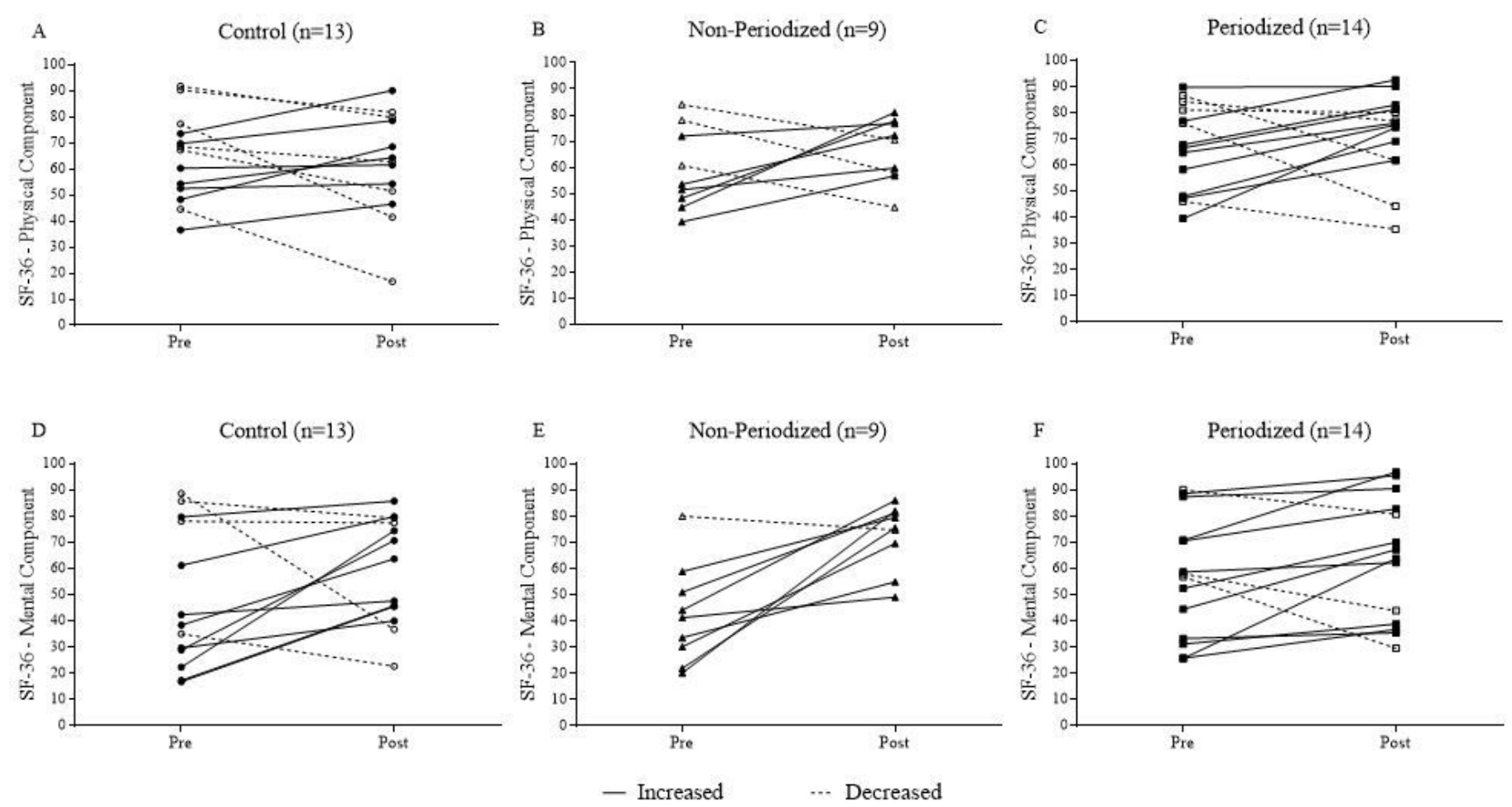

\section{Figure 2}

Individual scores in the components of health-related quality of life before and after the intervention period in adults with obesity.

\section{Supplementary Files}

This is a list of supplementary files associated with this preprint. Click to download.

- Table2.docx

- supplementarymaterial.docx 